\title{
Amentoflavone prevents sepsis-associated acute lung injury through Nrf2-GCLc-mediated upregulation of glutathione
}

\author{
Yuan Zong ${ }^{\bowtie}$ and Huali Zhang \\ Intensive Care Unit, Shaanxi Provincial People's Hospital, Xi'an 710068, Shaanxi Province, China
}

Sepsis is a serious medical problem and is one of the main causes of high mortality in intensive care units. Fifty percent of patients with severe sepsis will develop acute lung injury (ALI). Amentoflavone (AMF) is a polyphenolic compound possessing potent anti-inflammatory activities. The study aimed to explore the protective effects of AMF against ALI in cecal ligation and puncture (CLP)-induced septic rats. The results showed that AMF administration protected against septic ALI, as reflected by marked amelioration of histological injury of lung tissues and decrease of pulmonary edema in CLPtreated rats. AMF ameliorated CLP-induced increase of systemic and lung TNFa and IL-1 $\beta$ and binding activity of p65 NF-KB, indicating the inhibition of inflammation. Moreover, AMF prevented CLP-induced oxidative stress, as evidenced by increase of oxygen consumption rate, decrease of TBARS content, increase of SOD activity and GSH level in lung tissue of CLP-treated rats. CLP resulted in significant decrease of mRNA expression of Nrf2 and GCLC, which was inhibited by AMF. AMF-induced protective effects on $A L I$, inflammation, and oxidative stress were inhibited by lentivirus shRNA-mediated silence of Nrf2 and buthionine sulphoximine (BSO), an inhibitor of GSH synthesis. AMF increased Nrf2-binding activity in GCLc promoters in lung tissue of CLP-treated rats. The results suggested that AMF protected against ALI in septic rats through upregulation of Nrf2-GCLc signaling, enhancement of GSH antioxidant defense, reduction of oxidative stress and final amelioration of inflammation and histological injury of lung. The data provide new therapeutic options for the treatment of sepsis-associated ALI.

Key words: Sepsis; Acute lung injury; Amentoflavone; Nrf2; Glutathione

Received: 29 March, 2016; revised: 27 April, 2016; accepted: 04 May, 2016; available on-line: 08 October, 2016

\footnotetext{
e-mail: zongyuan1212@126.com

Abbreviations: ALI, acute lung injury; AMF, amentoflavone; ARDS, CHIP, chromatin immunoprecipitations acute respiratory distress syndrome; CLP, cecal ligation and puncture; GCLc, catalytic subunit of glutamate-cysteine ligase; GSH, gluthione; IL-1 $\beta$, interleukin- $1 \beta$; Nrf2, nuclear factor (erythroid-derived 2)-like 2; TBARS, thiobarbituric acid reactive substances; TNF-a, tumor necrosis factor- $a$
}

\section{INTRODUCTION}

Sepsis is a serious systemic inflammatory syndrome that is caused by infection, blood loss, trauma, and neoplasm (Liu et al., 2016). Sepsis affects millions of individuals worldwide and is one of the main causes of high mortality in intensive care units (ICU). To date, most of sepsis complications remain refractory, and development of novel effective therapeutic approaches is urgently needed. Although the mechanism of sepsis injury is not completely clear, oxidative stress has been believed to play a pivotal role (Bajcetic et al., 2014; Duran-Bedolla et al., 2014).

Sepsis could severely result in multiple organ dysfunction, including cardiovascular system, liver, kidney and lung. It is generally considered that damage of the lung is one of the most severe outcomes of sepsis (Andrews et al., 2005). It is estimated that about fifty percent of patients with severe sepsis will develop acute lung injury (ALI) and even acute respiratory distress syndrome (ARDS), a more severe consequence of ALI (Rubenfeld et al., 2005; Maybauer et al., 2006). During the development of ALI/ARDS, oxidative stress and inflammatory responses were enhanced, leading to the failure of multiple organs (Dolinay et al., 2012).

As a polyphenolic compound, amentoflavone (AMF) is derived from extracts of Selaginella tamariscina and has been found to possess potent anti-inflammatory activities (Huang et al., 2012; Tsai et al., 2012; Woo et al., 2005; Banerjee \& Valacchi et al., 2002). It is also found that AMF could inhibit the expression of cytokines in lung cell lines (Banerjee \& Van der Vliet et al., 2002). Banerjee showed that AMF inhibited TNF $\alpha$-induced cyclooxygenase- 2 expression through suppression of NF- $x \mathrm{~B}$ activation in A549 cells (Banerjee \& Van der Vliet et al., 2002). However, whether AMF exhibits beneficial effect on sepsis-induced ALI is not known.

Cecal ligation and puncture (CLP)-induced sepsis in animals is most frequently used as animal model of sepsis as it represents the characteristics of pathophysiology of human sepsis (Dejager et al., 2011; Scarlett et al., 2004). In the current study, using CLP-induced animal model of sepsis, we aimed to investigate the effect of AMF on sepsis-induced ALI, to focus on AMF-exhibited effect on inflammation and oxidative stress and to elucidate the possible mechanisms. The results showed that AMF prevented CLP-induced ALI through suppression of oxidative stress and inflammation via nuclear factor (erythroid-derived 2)-like 2 (Nrf2)/catalytic subunit of glutamate-cysteine ligase (GCLc)/gluthione (GSH) signaling.

\section{MATERIALS AND METHODS}

Chemicals and reagents. AMF and BSO were obtained from Sigma-Aldrich. TNF $\alpha$ and IL-1 $\beta$ ELISA kits were purchased from R\&D Systems. TBARS and SOD assay kits were obtained from Nanjing Jiancheng Company. GSH assay kit was obtained from Biovision. ELISA-based NF- $x \mathrm{~B}$ p65 transcription factor assay kit was purchased from Active Motif North America. CHIP as- 
say kit was obtained from Abcam. RNA isolation and Real-time PCR reagents were purchased from TaKaRa. All other chemicals used were of analytical grade.

Animals. Animal experiments were conducted under the guidelines for the care and use of laboratory animals published by the National Institute of Health. Experimental protocols were approved by the Ethical Committee of Shaanxi Provincial People's Hospital on Animal Care. Male Sprague-Dawley (SD) rats (220-260 g) were obtained from the Animal Center of Xi'an Jiaotong University. Rats were kept in a temperature-controlled room with a $12 \mathrm{~h}$ light/12 h dark cycle for 1 week before the experiment, with free access to food and water.

Animal model of sepsis. Fifty animals were randomly divided into five groups (10 rats in each group): Sham group, CLP group, AMF group (CLP + $50 \mathrm{mg}$ / $\mathrm{kg} \mathrm{AMF}), \mathrm{AMF}+\mathrm{LV}-\mathrm{shNrf2}(\mathrm{CLP}+50 \mathrm{mg} / \mathrm{kg}$ AMF + LV-shRNA Nrf2), AMF + BSO (CLP + $50 \mathrm{mg} / \mathrm{kg}$ $\mathrm{AMF}+10 \mathrm{mg} / \mathrm{kg}$ buthionine sulfoximine). CLP-induced sepsis model was built in CLP, AMF, AMF + LV-shNrf2, and AMF + BSO groups as previously described (Liu et al., 2016). In brief, after fasting overnight, rats were anesthetized using $10 \%$ chloral hydrate $(3 \mathrm{ml} /$ $\mathrm{kg}$, intraperitoneal). After sterilization, a $1.5-\mathrm{cm}$ incision was cut in ventral midline of abdomen and then the cecum was gently exposed and ligated with a 3-0 silk and punctured with an 18-gauge needle at three locations. After that, the cecum was repositioned and the abdomen was closed. The same sham surgery and cecum manipulation was conducted in Sham rats without ligation and puncture. Rats in AMF + LV-shNrf2 group were treated intranasally with either $4 \times 10^{7}$ PFUs (in $50 \mu \mathrm{l}$ ) letivirus shRNA targeting Nrf2 (Shanghai GenePharma Co., Ltd, China) 24 hours before the surgery and rats in the other groups were treated with scramble shRNAs. Rats in $\mathrm{AMF}+\mathrm{BSO}$ group were intraperitoneally injected with $10 \mathrm{mg} / \mathrm{kg}$ BSO 2 hours before the surgery and rats in the other groups were injected with vehicle. Rats in AMF, AMF + LV-shNrf2, and AMF + BSO groups were intraperitoneally administrated with $50 \mathrm{mg} /$ $\mathrm{kg}$ AMF 6 hours before the surgery and rats in the other groups were given with vehicle $(0.1 \%$ Tween 80$)$. Saline ( $2 \mathrm{ml} / 100 \mathrm{~g}$ body weight) was given subcutaneously to the rats immediately after the operation for resuscitation. No antibiotics were administered. AMF was administrated daily for 5 consecutive days. 6 days after the surgery, rats were euthanized. Blood samples were collected. Left lung was excised for wet/dry ratio analysis. Part of right lung was excised for histological examination. Part of right lung was homogenized in a phosphate buffer solution for further biochemical analysis. Part of right lung was stored at $-70^{\circ} \mathrm{C}$ for real-time PCR detection.

Histological examination. Lung tissues were fixed in 4\% paraformaldehyde overnight, dehydrated, embedded, and sliced into $5-\mu \mathrm{m}$ sections. After that, sections were stained with hematoxylin and eosin, and were observed under a light microscopy (Olympus, Tokyo, Japan).

Lung wet/dry weight ratio. Wet weight of left lung was recorded immediately after the excision. Then the lung tissues were placed in an incubator at $70^{\circ} \mathrm{C}$ for $48 \mathrm{~h}$ and the dry weight was recorded. The wet to dry weight ratio was calculated as follows: wet weight-dry weight/dry weight.

Determination of inflammation. Levels of tumor necrosis factor-alpha (TNF $\alpha$ ) and interleukin-1 $\beta$ (IL-1 $\beta$ ) in serum and lung homogenates were determined by ELISA kits according to the manufacturer's instructions. Nuclear protein was extracted and DNA-binding activity of p $65 \mathrm{NF}-x \mathrm{~B}$ was determined using an ELISA-based
NF- $x$ B p 65 transcription factor assay kit according to the manufacturer's protocols. In each assay, a standard curve and positive/negative controls were included. The absorbance at $450 \mathrm{~nm}$ was measured using a microtiter ELISA reader (BioRad, USA). The assay sensitivity for $\mathrm{IL}-1 \beta$ was $<2 \mathrm{pg} / \mathrm{mL}$ and for $\mathrm{TNF}-\alpha$ was $0.5-5.5 \mathrm{pg} /$ $\mathrm{mL}$.

Oxygen consumption rate. Mitochondrial function in lung tissue was assessed by the rate of oxygen consumption. In brief, mitochondria were isolated from lung tissues according to the manufacture's protocols (Thermo scientific, USA). Mitochondria were isolated using differential centrifugation procedures. The resulting pellets were suspended in Tris buffer and mitochondrial marker cytochrome $\mathrm{c}$ was detected to evaluate the purity of mitochondria. A Clark-type Oxygen Electrode was used to measure oxygen consumption rate. Respiration was initiated by addition of $2 \mathrm{mM}$ pyruvate $+5 \mathrm{mM}$ malate or $5 \mathrm{mM}$ succinate. After $2 \mathrm{~min}$, state 3 respiration was induced by adding $1 \mathrm{mM}$ ADP. The results were expressed as percentage of oxygen consumption.

Determination of oxidative stress. TBARS levels and SOD activities in lung homogenates were detected by commercial kits (Nanjing Jiancheng Company, China). TBARS content was determined by the TBA reaction. The reaction mixture was heated at constant temperature at $95^{\circ} \mathrm{C}$ for $40 \mathrm{~min}$, cooled by running water and then centrifuged at $3500 \mathrm{r} / \mathrm{min}$ for $10 \mathrm{~min}$. The absorbance at $532 \mathrm{~nm}$ was measured and the results were shown as $\mu \mathrm{mol} / \mathrm{mg}$ protein. SOD activity was measured through detection of nitroblue tetrazolium reduction at $450 \mathrm{~nm}$. One unit of SOD activity was defined as the amount of enzyme required to inhibit the oxidation reaction by $50 \%$ and was expressed as $\mathrm{U} / \mathrm{mg}$ protein. GSH content in lung homogenates was determined by a commercial assay kit (Biovision, USA). GSH was detected by the specific reaction of o-phthalaldehyde with GSH (not GSSG). The fluorescence of the product was measured at Ex/Em 340/420 nm. The results were shown as $\mathrm{nmol} / \mathrm{mg}$ protein.

Real-time PCR. Total RNA was isolated from lung tissues using the TRIzol reagent. 500 ng total RNA was transcribed into cDNA using a commercial kit (TaKa$\mathrm{Ra}$, Japan) according to the manufacturer's instructions. Real-time PCR was performed in a $20 \mu$ l reaction mixture containing $10 \mu \mathrm{l} 2 \times$ SYBR green master mix, $7 \mu \mathrm{l}$ $\mathrm{H}_{2} \mathrm{O}, 2 \mu \mathrm{l}$ primers $(10 \mu \mathrm{M})$, and $1 \mu \mathrm{l}$ template DNA. Cycling was conducted using a quantitative PCR instrument (BIORAD, USA) and a SYBR ${ }^{\circledR}$ Premix Ex Taq ${ }^{\text {TM }}$ kit (TaKaRa, Japan) were used to detect target gene expressions, and $\beta$-actin was used as an internal reference. The $2^{-\Delta \Delta C T}$ method was employed to determine the relative expression of target genes normalized to $\beta$-actin, and experiments were repeated in triplicate.

Chromatin immunoprecipitations. Chromatin immunoprecipitations (CHIP) was conducted to evaluate the transcriptional activity of $\mathrm{Nrf} 2$ in the regulation of GCLc. Chromatin of tissues prepared according to instructions of a kit for chromatin preparation from tissues for ChIP (Abcam, USA). Immunoprecipitations were carried out using anti-Nrf2 antibody (Cell Signaling Technology, USA) or normal mouse IgG (Abcam). Real-time PCR was performed and the results were normalized to the respective input signals and expressed as folds of sham.

Statistical analysis. Results were expressed as means \pm S.E.M. Graph-Pad Prism software was used to perform the statistical analysis using one-way ANOVA followed by Newmane Keuls multiple-comparison post hoc 

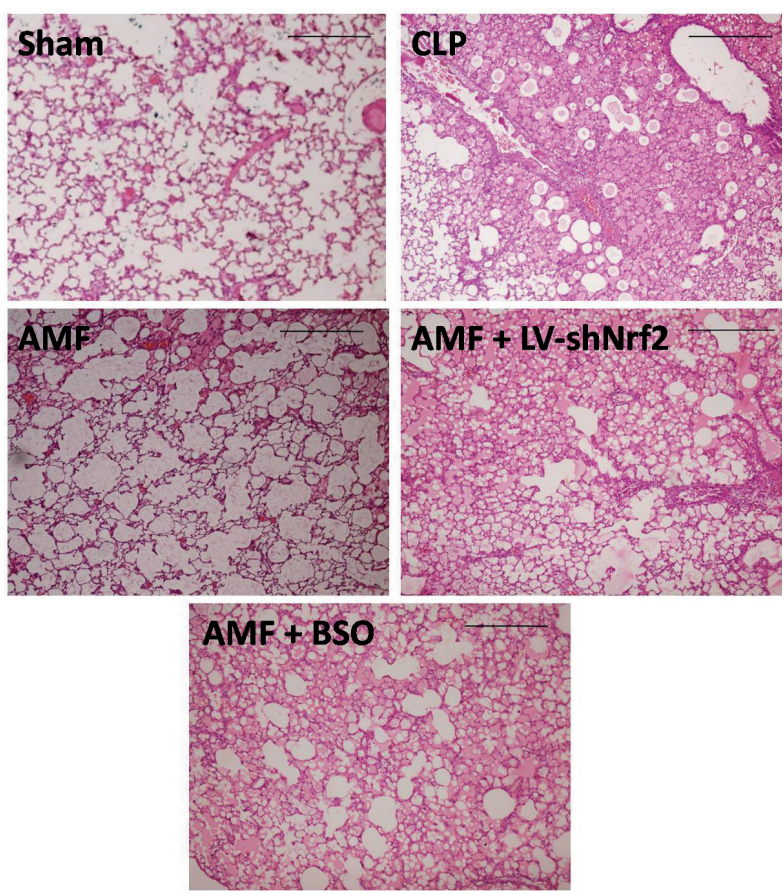

Figure 1. Effect of AMF on histological injury of lung tissue in CLP-treated rats.

CLP-induced sepsis model was built in rats and some rats were pre-administrated with AMF or LV-shNrf2 or BSO before the surgery. After the treatment, histological injury of lung tissues was measured by HE staining.

test. $P$ value $<0.05$ was considered to be statistically significant.

\section{RESULTS}

\section{AMF ameliorated CLP-induced histological injury in lung tissues}

To evaluate the effect of AMF on CLP-induced injury in lung tissues in rats, histological examination was performed. As shown in Fig. 1, we showed that there was severe hemorrhage, alveolar congestion, thickening of alveolar wall, and infiltration and aggregation of neutrophils in alveoli and vessel walls in the lung of CLPtreated mice, indicating that CLP resulted in significant inflammatory response. The administration of AMF notably inhibited these inflammatory changes in rats. However, the treatment of letivirus of shNrf2 or BSO, a glutathione (GSH) synthesis inhibitor, markedly inhibited AMF-induced improvement of inflammatory response in lung tissues.

\section{AMF ameliorated CLP-induced pulmonary edema}

Wet/dry weights ratio of lung tissue was detected. In Fig. 2, we show that CLP induced a significant increase of wet/dry weights ratio of lung tissue, indicating that CLP resulted in a marked pulmonary edema. AMF administration markedly inhibited the increase of wet/dry weights ratio of lung tissue in CLP-treated rats. In contrast, the treatment of shNrf2 letivirus or BSO markedly suppressed AMF-induced amelioration of pulmonary edema in CLP-treated rats.

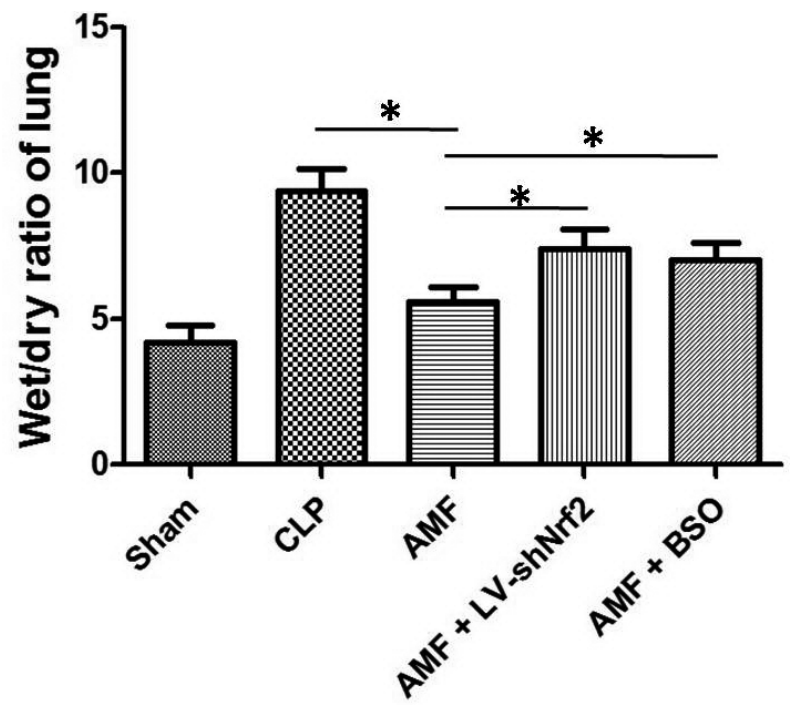

Figure 2. Effect of AMF on wet/dry weights of lung tissue in CLP-treated rats.

After the treatment, wet and dry weights of lung tissues were weighed and wet/dry raio of lung tissue was calculated $(p=0.0002)$. ${ }^{*} p<0.05$, statistical difference between the two groups.

\section{AMF ameliorated CLP-induced inflammation}

To evaluate the effect of AMF on inflammation in CLP-treated rats, levels of cytokines and activity of p65 NF- $x \mathrm{~B}$ were measured. Systemic and lung levels of $\mathrm{TNF} \alpha$ and IL-1 $\beta$ were determined by ELISA kits and the results showed that serum and lung TNF $\alpha$ and IL$1 \beta$ levels were significantly increased by CLP (Fig. 3A_C and D). Moreover, p65 NF- $x \mathrm{~B}$ binding activity was markedly increased by CLP (Fig. 3E). AMF administration markedly inhibited CLP-induced increase of serum and lung TNF $\alpha$ and IL-1 $\beta$ levels and p65 NF- $x \mathrm{~B}$ binding activity (Fig. 3). shNrf2 letivirus or BSO treatment could suppress AMF-induced inhibition of serum and lung TNF $\alpha$ and IL-1 $\beta$ levels and p 65 NF- $x$ B binding activity in CLP-treated rats (Fig. 3). The results indicated that AMF administration prevented CLP-induced inflammation in lung tissue which was inhibited by shNrf2 or BSO.

\section{AMF ameliorated CLP-induced oxidative stress}

To evaluate the effect of AMF on oxidative stress in rats treated by CLP, mitochondrial function and several oxidation-sensitive markers were determined. Mitochondria are main targets of oxidative injury and mitochondrial function is a sensitive indicator of oxidative stress status. In Fig. 4A, we showed that oxygen consumption rate in lung tissues treated by CLP was significantly decreased. AMF markedly increased oxygen consumption rate in lung tissues of CLP-treated rats (Fig. 4A). AMF-induced effect on mitochondrial function was inhibited by knockdown of $\mathrm{Nrf} 2$ and the treatment of BSO (Fig. 4A). As shown in Fig. 4B, C and D, CLP resulted in a significant increase of thiobarbituric acid reactive substances (TBARS) content, a marked decrease of superoxide dismutase (SOD) activities and GSH levels in lung tissues. AMF markedly prevented CLP-induced changes of oxidative stress markers in lung tissues which effect was inhibited by shRNA of Nrf2 and BSO (Fig. $4 \mathrm{~B}, \mathrm{C}$ and $\mathrm{D})$. The results demonstrated that AMF administration prevented CLP-induced oxidative injury in 

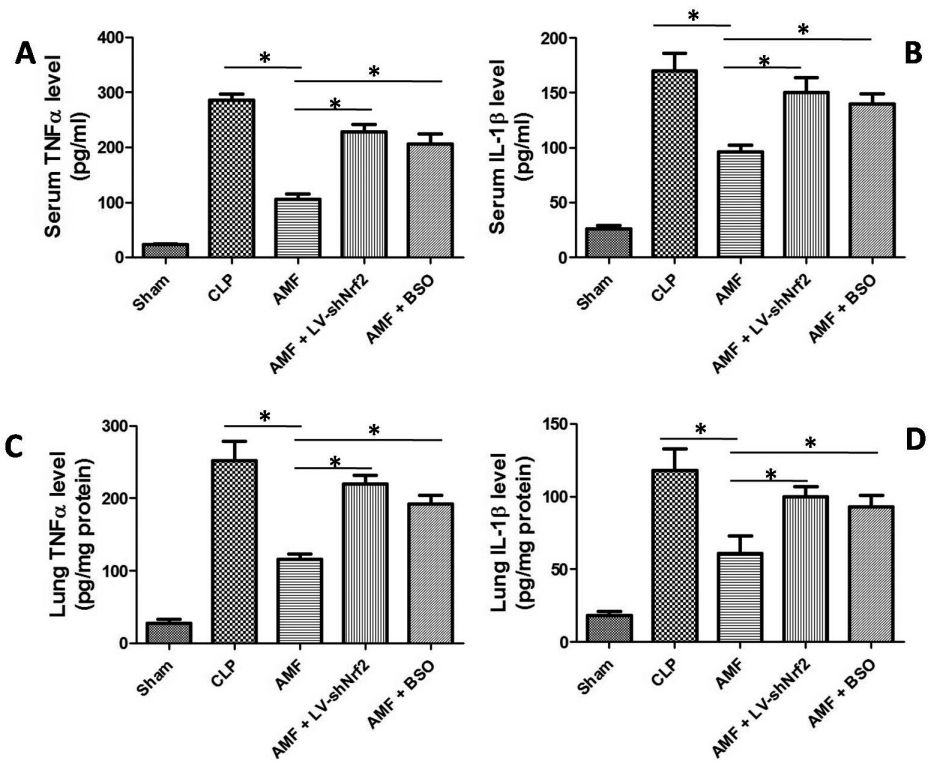

E

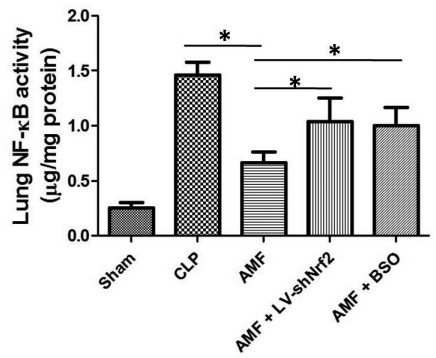

Figure 3. Effect of AMF on inflammation in lung tissue in CLP-treated rats.

Serum $(A$ and $B$ ) and lung (C and D) TNFa (A and $C$ ) and IL-1 $\beta$ (B and $D)$ levels were detected using commercial ELISA kits. Results were expressed as $\mathrm{pg} / \mathrm{ml}$ or $\mathrm{pg} / \mathrm{mg}$ protein. $P<0.001$ in these experiments. (E) p65 NF-KB binding activity was determined by an ELISA kit. Results were expressed as $\mu \mathrm{g} / \mathrm{mg}$ protein $(p<0.001)$. ${ }^{*} p<0.05$, statistical difference between the two groups.
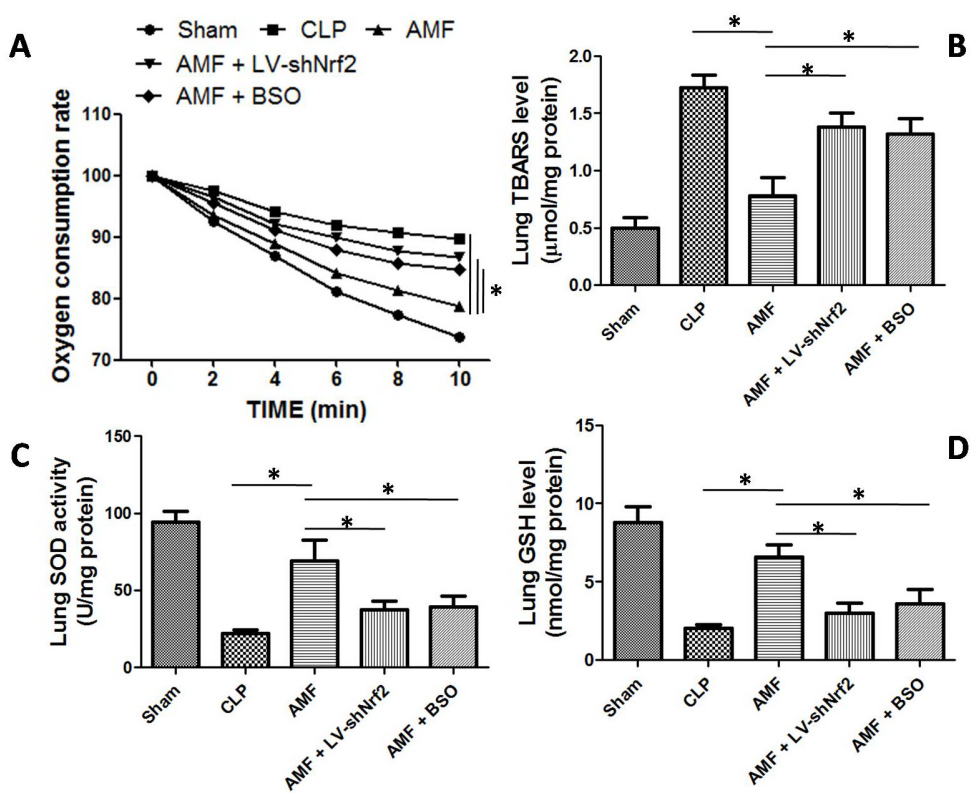

Figure 4. Effect of AMF on oxidative stress in lung tissue in CLP-treated rats.

After the treatment, (A) mitochondria in lung tissue were isolated and oxygen consumption rate was measured. Results were shown as percentage of oxygen consumption rate $(p=0.0003)$. TBARS content $(B)$, SOD activity $(C)$ and GSH level (D) in lung tissue homogenates were determined by commercial assay kits. Results were expressed as $\mu \mathrm{mol} / \mathrm{mg}$ protein $(p<0.001), \mathrm{U} / \mathrm{mg}$ protein $(p<0.001)$ and $\mathrm{nmol} / \mathrm{mg}$ protein $(p<0.001)$, respectively. ${ }^{*} p<0.05$, statistical difference between the two groups. lung tissue and shNrf2 and BSO could inhibit the antioxidative effect of AMF.

\section{AMF prevented CLP-induced decrease of Nrf2-GCLc signaling}

Considering the pivotal role of GSH in the antioxidant defense system, we further explored the mechanism of AMF-induced regulation of GSH. Expression of GCLc, the rate-limiting enzyme for GSH synthesis, was determined. As shown in Fig. 5A, mRNA expression of GCLc was significantly decreased by CLP. AMF markedly inhibited CLP-induced decrease of GCLc mRNA expression (Fig. 5A). shNrf2, but not $\mathrm{BSO}$, resulted in a significant inhibition of AMF-exhibited suppression of CLP-induced decrease of GCLc mRNA expression (Fig. 5A). To further explore the possible mechanism underlying AMF-induced regulation of GCLc expression, expression and activity of $\mathrm{Nrf2}$, a central transcription factor controlling redox balance, were measured. In Fig. 5B, we showed that AMF induced a significant inhibition of CLP-resulted decrease of Nrf2 mRNA expression. The effect of AMF on Nrf2 mRNA expression in CLP-treated rats was suppressed by shRNA of $\mathrm{Nrf} 2$, but not BSO (Fig. 5B). Moreover, CLP resulted in a notable decrease of Nrf2binding in GCLc promoters which effect was prevented by AMF administration (Fig. 5C). shRNA of Nrf2, but not BSO, suppressed AMF-induced increase in Nrf2-binding activity in GCLc promoters (Fig. 5C). The results demonstrated that regulation of GCLc by $\mathrm{Nrf2}$ may be involved in AMF-induced protective effects against CLP-resulted GSH depletion and lung injury.

\section{DISCUSSION}

CLP-induced sepsis model is a widely accepted animal model for the research of sepsis-associated mechanism and the investigation of potential therapeutic options for the treatment of sepsis. In the current study, using CLP-induced septic rats, we investigated the protective effects of AMF against sepsisassociated ALI. We found that AMF administration protected against septic ALI, as reflected by marked amelioration of histological injury of lung tissues and decrease of pulmonary edema in CLP-treated rats.

It is generally believed that inflammation is the fundamental pathological process in the pathogenesis of organ injury in sepsis (Dounousi et al., 2016; Hahn et al., 2016). Especially for ALI, inhibition of inflammation is considered to be a potent therapeutic strategy (Xie et al., 2012; Lin et al., 2015). It has been found 


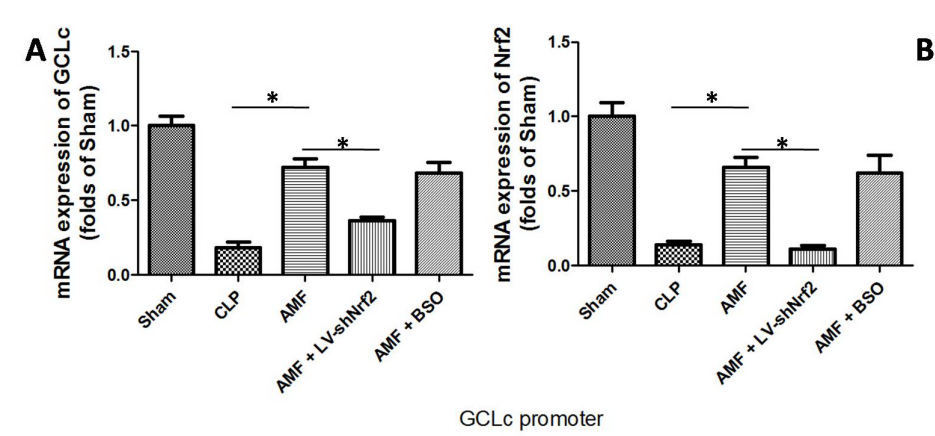

C

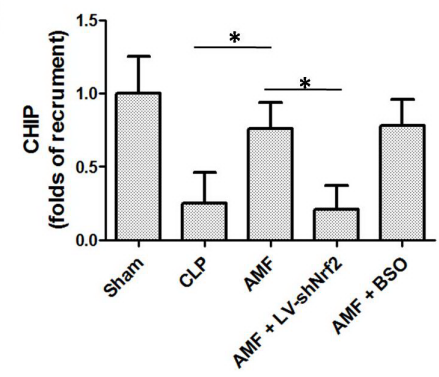

Figure 5. Effect of AMF on the regulation of GCLc by Nrf2 in lung tissue in CLPtreated rats.

mRNA expression of GCLc (A) and Nrf2 (B) in lung tissues was determined by real-time PCR. Results were shown as folds of sham group. (A) $p=0.003 ;(\mathbf{B}) p=0.002$. The binding activity of Nrf2 to GCLc promoters was determined by CHIP assay (C) and results were shown as folds of sham $(p=0.007)$. ${ }^{*} p<0.05$, statistical difference between the two groups.

that proinflammatory cytokines, such as TNF $\alpha$ and IL-1 $\beta$, are increased in the serum of septic patients (Kang et al., 2001a). NF- $x$ B is a central transcription factor in the regulation of inflammation through controlling various proinflammatory gene expression, including TNF $\alpha$, IL-1 $\beta, \mathrm{IL}-6$, and IL-8 (Cortez et al., 2013). NF- $x$ B-mediated inflammation plays an important role in the pathogenesis of ALI and sepsis (Kang et al., 2001b). p65 is a major sub-member of NF- $x \mathrm{~B}$ that mediates its proinflammatory effect. Consistent with previous literature, we found that CLP resulted in severe inflammation, as illustrated by increase of systemic and lung TNF $\alpha$ and IL- $1 \beta$ levels and the binding activity of NF- $x \mathrm{~B}$. The administration of AMF significantly inhibited these changes induced by CLP, indicating the amelioration of inflammation.

To explore the mechanism of the protective effect of AMF against CLP-induced inflammation and ALI, redox status was measured in the study. We found that AMF administration notably inhibited CLP-induced decrease of oxygen consumption, increase of TBARS content, decrease of SOD activity and GSH level, indicating that AMF inhibited CLP-induced oxidative stress. Oxidative stress is defined as imbalance between reactive oxygen species (ROS) generation and antioxidant defense (Avogaro et al., 2008). Damage of antioxidant system could result in excessive accumulation of ROS, leading to oxidative injury of various macromolecules and important organelles (Avogaro et al., 2008). Oxidative stress is closely related with inflammation and contributes to sepsis-associated injury (Zolali et al., 2015; Gerin et al., 2016). Mitochondrial function is a sensitive indicator of intracellular redox state. TBARS indicates the content of malondialdehyde (MDA), an end product of lipid peroxidation (Napierala et al., 2016). SOD is an important antioxidant enzyme catalyzing the dismutase of superoxide into hydrogen peroxide (Shi et al., 2016). GSH is one of the most important antioxidant proteins, functioning to eliminate ROS directly or indirectly under the catalysis of antioxidant enzymes (Couto et al., 2016). We should point out that the markers of oxidative stress determined in the study, including TBARS, SOD, and GSH, are indirect markers. Only the antioxidant enzyme SOD was determined to evaluate the antioxidant defense. In future study, more direct markers, such as ROS concentration, and antioxidant enzymes are needed to accurately evaluate the redox status. Even so, based on previous literature and our results, we demonstrated that the antioxidative activity of AMF was involved in the protective effects against sepsis-associated ALI.

To further explore the molecular mechanism of AMF-exhibited antioxidative activity, regulation of GSH synthesis by AMF was evaluated. GCLc is reported to be the rate-limiting enzyme for the synthesis of GSH under the transcriptional regulation of Nrf2 (Jiang et al., 2016; Takahashi et al., 2016; Kuwano et al., 2015). Activation of Nrf2 and its target gene GCLc was show to protect against LPS-induced inflammatory response (Thimmulappa et al., 2007; Thimmulappa et al., 2006). In the present study, we showed that knockdown of Nrf2 by lentivirus and inhibition of GSH synthesis by BSO significantly suppressed AMF-induced inhibitory effect on histological injury, pulmonary edema, inflammatory response and oxidative stress in the development of ALI in septic rats. We also showed that AMF increased the binding activity of $\mathrm{Nrf2}$ in the promoters of GCLc. The results demonstrated that enhancement of GSH through Nrf2-regulated GCLc was involved in the protective effects of AMF. Further studies are needed to explore whether Nrf2 is a direct target of AMF and to examine the structure-activity relationship. Since the mRNA expression of Nrf2 was altered, it was indicated that AMF could activate the upstream transcriptional regulator of $\mathrm{Nrf2}$ which needed further efforts to elucidate. Moreover, the results also showed that knockdown of Nrf2 and inhibition of GSH synthesis did not completely reversed the beneficial effect of AMF, indicating that additional mechanism may be involved in the protective effect of AMF against sepsis. Furthermore, we should notice that AMF could not completely prevent sepsis-associated injury. Therefore, AMF can be used as a promising therapeutic option in combination with additional agents in the prevention and treatment of sepsis and its complications.

In conclusion, in the current study, we found that AMF protected against ALI in septic rats through upregulation of Nrf2-GCLc signaling, enhancement of GSH antioxidant defense, reduction of oxidative stress and final amelioration of inflammation and histological injury of lung. The data provide new therapeutic options for the treatment of sepsis-associated ALI.

\section{Conflict of interest}

The authors declare none.

\section{REFERENCES}

Andrews P, Azoulay E, Antonelli M, Brochard L, Brun-Buisson C, Dobb G, Fagon JY, Gerlach H, Groeneveld J, Mancebo J, Metnitz 
P, Nava S, Pugin J, Pinsky M, Radermacher P, Richard C, Tasker $\mathrm{R}$, Vallet B (2005) Year in review in intensive care medicine, 2004. I. Respiratory failure, infection, and sepsis. Intensive Care Med 31: 28-40. DOI: $10.1007 / \mathrm{s} 00134-004-2529-5$

Avogaro A, de Kreutzenberg SV, Fadini GP (2008) Oxidative stress and vascular disease in diabetes: is the dichotomization of insulin signaling still valid? Free Radic Biol Med 44: 1209-1215. DOI: 10.1016/j.freeradbiomed.2007.12.021

Bajcetic M, Spasic S, Spasojevic I (2014) Redox therapy in neonatal sepsis: reasons, targets, strategy, and agents. Shock 42: 179-184. DOI: $10.1097 /$ SHK.0000000000000198

Banerjee T, Valacchi G, Ziboh VA, van der Vliet A (2002) Inhibition of TNFalpha-induced cyclooxygenase- 2 expression by amentoflavone through suppression of NF-kappaB activation in A549 cells. Mol Cell Biochem 238: 105-110. DOI: 10.1023/A:1019963222510

Banerjee T, Van der Vliet A, Ziboh VA (2002) Downregulation of COX-2 and iNOS by amentoflavone and quercetin in A549 human lung adenocarcinoma cell line. Prostaglandins Leukot Essent Fatty Acids 66: 485-492. DOI: 10.1054/plef.2002.0387

Cortez M, Carmo LS, Rogero MM, Borelli P, Fock RA (2013) A highfat diet increases IL-1, IL- 6 , and TNF- $\alpha$ production by increasing $\mathrm{NF}-x \mathrm{~B}$ and attenuating PPAR- $\gamma$ expression in bone marrow mesenchymal stem cells. Inflammation 36: 379-386. DOI: 10.1007/s10753$012-9557-z$

Couto N, Wood J, Barber J (2016) The role of glutathione reductase and related enzymes on cellular redox homoeostasis network. Free Radic Biol Med 95: 27-42. DOI: 10.1016/j.freeradbiomed.2016.02.028

Dejager L, Pinheiro I, Dejonckheere E, Libert C (2011) Cecal ligation and puncture: the gold standard model for polymicrobial sepsis? Trends Microbiol 19: 198-208. DOI: 10.1016/j.tim.2011.01.001

Dolinay T, Kim YS, Howrylak J, Hunninghake GM, An CH, Fredenburgh L, Massaro AF, Rogers A, Gazourian L, Nakahira K, Haspel JA, Landazury R, Eppanapally S, Christie JD, Meyer NJ, Ware LB, Christiani DC, Ryter SW, Baron RM, Choi AM (2012) Inflammasome-regulated cytokines are critical mediators of acute lung injury. Am J Respir Crit Care Med 185: 1225-1234. DOI: 10.1164/ rccm.201201-0003OC

Dounousi E, Torino C, Pizzini P, Cutrupi S, Panuccio V, D’Arrigo G, Abd ElHafeez S, Tripepi G, Mallamaci F, Zoccali C'(2016) Effect of inflammation by acute sepsis on intact fibroblast growth factor 23 (iFGF23) and asymmetric dimethyl arginine (ADMA) in CKD patients. Nutr Metab Cardiovasc Dis 26: 80-83. DOI: 10.1016/j.numecd.2015.10.004

Duran-Bedolla J, Montes DOM, Saldana-Navor V, Villalobos-Silva JA, Rodriguez MC, Rivas-Arancibia S (2014) Sepsis, mitochondrial failure and multiple organ dysfunction. Clin Invest Med 37: E58-E69

Gerin F, Sener U, Erman H, Yilmaz A, Aydin B, Armutcu F, Gurel A (2016) The effects of quercetin on acute lung injury and biomarkers of inflammation and oxidative stress in the rat model of sepsis. Inflammation 39: 700-705. DOI: 10.1007/s10753-015-0296-9

Hahn WO, Mikacenic C, Price BL, Harju-Baker S, Katz R, Himmelfarb J, Wurfel MM, Liles WC (2016) Host derived biomarkers of inflammation, apoptosis, and endothelial activation are associated with clinical outcomes in patients with bacteremia and sepsis regardless of microbial etiology. Virulence DOI: 10.1080/21505594.2016.1144003.

Huang N, Rizshsky L, Hauck CC, Nikolau BJ, Murphy PA, Birt DF (2012) The inhibition of lipopolysaccharide-induced macrophage inflammation by 4 compounds in Hypericum perforatum extract is partially dependent on the activation of SOCS3. Phytochemistry 76: 106-116. DOI: 10.1016/j.phytochem.2011.12.001

Jiang YM, Wang Y, Tan HS, Yu T, Fan XM, Chen P, Zeng H, Huang M, Bi HC (2016) Schisandrol B protects against acetaminopheninduced acute hepatotoxicity in mice via activation of the NRF2/ ARE signaling pathway. Acta Pharmacol Sin 37: 382-389. DOI: 10.1038/aps.2015.120

Kang JL, Lee HW, Lee HS, Pack IS, Chong Y, Castranova V, Koh Y (2001a) Genistein prevents nuclear factor-kappa B activation and acute lung injury induced by lipopolysaccharide. Am J Resp Crit Care 164: $2206-2212$
Kang JL, Lee HW, Lee HS, Pack IS, Chong Y, Castranova V, Koh Y (2001b) Genistein prevents nuclear factor-kappa B activation and acute lung injury induced by lipopolysaccharide. Am J Respir Crit Care Med 164: 2206-2212. DOI: 10.1164/ajrccm.164.12.2104017

Kuwano T, Watanabe M, Kagawa D, Murase T (2015) Hydrolyzed methylhesperidin induces antioxidant enzyme expression via the Nrf2-ARE pathway in normal human epidermal keratinocytes. $J A g$ ric Food Chem 63: 7937-7944. DOI: 10.1021/acs.jafc.5b01992

Lin WC, Chen CW, Huang YW, Chao L, Chao J, Lin YS, Lin CF (2015) Kallistatin protects against sepsis-related acute lung injury via inhibiting inflammation and apoptosis. Sci Rep 5: 12463. DOI: $10.1038 /$ srep 12463

Liu CH, Zhang WD, Wang JJ, Feng SD (2016) Senegenin ameliorate acute lung injury through reduction of oxidative stress and inhibition of inflammation in cecal ligation and puncture-induced sepsis rats. Inflammation 39: 900-906. DOI: 10.1007/s10753-016-0322-6

Napierala M, Merritt TA, Mazela J, Jablecka K, Miechowicz I, Marszalek A, Florek E (2016) The effect of tobacco smoke on oxytocin concentrations and selected oxidative stress parameters in plasma during pregnancy and post-partum - an experimental model. Hum Exp Toxicol DOI: 10.1177/0960327116639363

Rubenfeld GD Caldwell E Peabody E, Weaver J, Martin DP, Neff M, Stern EJ, Hudson LD (2005) Incidence and outcomes of acute lung injury. $N$ Engl J Med 353: 1685-1693. DOI: 10.1056/NEJMoa050333

Scarlett CJ, O'Leary MJ, Kee AJ, Nielsen A, Sevette A, Baxter RC, Smith RC (2004) A study of parenteral versus enteral nutrition following caecal ligation and puncture in the rat: Influence on survival and tissue protein turnover. Clin Nutr 23: 1135-1145. DOI: 10.1016/j.clnu.2004.02.008

Shi H, Shi A, Dong L, Lu X, Wang Y, Zhao J, Dai F, Guo X (2016) Chlorogenic acid protects against liver fibrosis in vivo and in vitro through inhibition of oxidative stress. Clin Nutr DOI: 10.1016/j. clnu.2016.03.002. DOI: 10.1016/j.clnu.2016.03.002

Takahashi S, Hisatsune A, Kurauchi Y, Seki T, Katsuki H (2016) Insulin-like growth factor 1 specifically up-regulates expression of modifier subunit of glutamate-cysteine ligase and enhances glutathione synthesis in SH-SY5Y cells. Eur J Pharmacol 771: 99-106. DOI: 10.1016/j.ejphar.2015.12.013

Thimmulappa RK, Fuchs RJ, Malhotra D, Scollick C, Traore K, Bream JH, Trush MA, Liby KT, Sporn MB, Kensler TW, Biswal S (2007) Preclinical evaluation of targeting the $\mathrm{Nrf} 2$ pathway by triterpenoids (CDDO-Im and CDDO-Me) for protection from LPS-induced inflammatory response and reactive oxygen species in human peripheral blood mononuclear cells and neutrophils. Antioxid Redox Signal 9: 1963-1970. DOI: 10.1089/ars.2007.1745

Thimmulappa RK, Scollick C, Traore K, Yates M, Trush MA, Liby KT, Sporn MB, Yamamoto M, Kensler TW, Biswal S (2006) Nrf2dependent protection from LPS induced inflammatory response and mortality by CDDO-Imidazolide. Biochem Biophys Res Commun 351: 883-889. DOI: 10.1016/j.bbrc.2006.10.102

Tsai SC, Liang YH, Chiang JH, Liu FC, Lin WH, Chang SJ, Lin WY, Wu CH, Weng JR (2012) Anti-inflammatory effects of Calophyllum inophyllum L. in RAW264.7 cells. Oncol Rep 28: 1096-1102. DOI: 10.3892/or.2012.1873

Woo ER, Lee JY, Cho IJ, Kim SG, Kang KW (2005) Amentoflavone inhibits the induction of nitric oxide synthase by inhibiting NFkappaB activation in macrophages. Pharmacol Res 51: 539-546. DOI: 10.1016/j.phrs.2005.02.002

Xie K, Yu Y, Huang Y, Zheng L, Li J, Chen H, Han H, Hou L, Gong G, Wang G (2012) Molecular hydrogen ameliorates lipopolysaccharide-induced acute lung injury in mice through reducing inflammation and apoptosis. Shock 37: 548-555. DOI: 10.1097/ SHK.0b013e31824ddc81

Zolali E, Asgharian P, Hamishehkar H, Kouhsoltani M, Khodaii H, Hamishehkar H (2015) Effects of gamma oryzanol on factors of oxidative stress and sepsis-induced lung injury in experimental animal model. Iran J Basic Med Sci 18: 1257-1263 